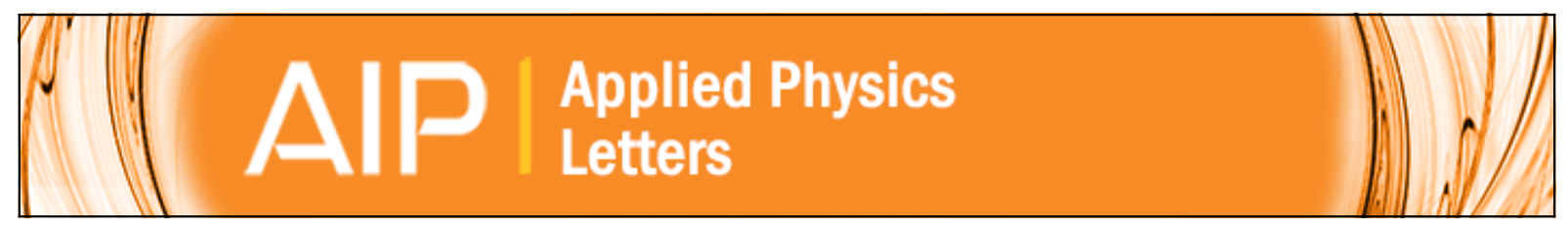

\title{
Evolution of optical phonons in CdS nanowires, nanobelts, and nanosheets
}

Kyoung-Yeon Lee, Jung-Ran Lim, Heesuk Rho, Young-Jin Choi, Kyoung Jin Choi, and Jae-Gwan Park

Citation: Applied Physics Letters 91, 201901 (2007); doi: 10.1063/1.2806937

View online: http://dx.doi.org/10.1063/1.2806937

View Table of Contents: http://scitation.aip.org/content/aip/journal/apl/91/20?ver=pdfcov

Published by the AIP Publishing

\section{Articles you may be interested in}

Enhanced broadband photoresponse of $\mathrm{Ge} / \mathrm{CdS}$ nanowire radial heterostructures

Appl. Phys. Lett. 94, 223119 (2009); 10.1063/1.3149704

In situ growth of aligned CdS nanowire arrays on Cd foil and their optical and electron field emission properties

J. Appl. Phys. 104, 014312 (2008); 10.1063/1.2952013

Anisotropy of electron-phonon coupling in single wurtzite CdS nanowires

Appl. Phys. Lett. 91, 171911 (2007); 10.1063/1.2805380

Admittance of CdS nanowires embedded in porous alumina template

Appl. Phys. Lett. 88, 113114 (2006); 10.1063/1.2185729

Resonant Raman scattering from CdS nanowires

Appl. Phys. Lett. 88, 043118 (2006); 10.1063/1.2168507

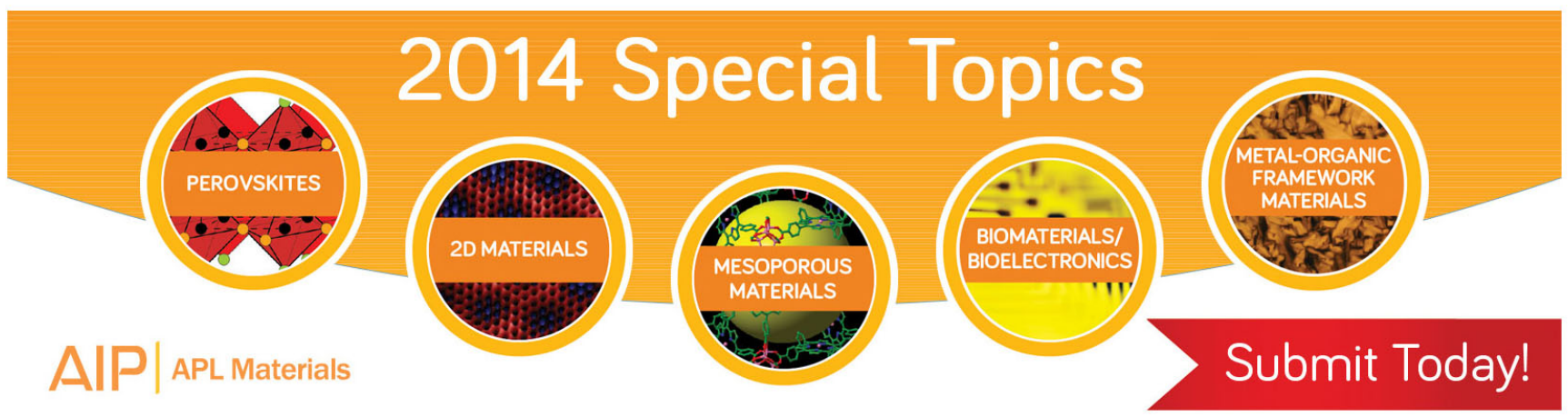




\title{
Evolution of optical phonons in CdS nanowires, nanobelts, and nanosheets
}

\author{
Kyoung-Yeon Lee, Jung-Ran Lim, and Heesuk Rho a) \\ Department of Physics, Chonbuk National University, Jeonju 561-756, Korea \\ Young-Jin Choi, Kyoung Jin Choi, and Jae-Gwan Park \\ Nano Materials Research Center, Korea Institute of Science and Technology, Seoul 130-650, Korea
}

(Received 1 July 2007; accepted 17 October 2007; published online 12 November 2007)

\begin{abstract}
We report Raman scattering from single and ensemble CdS nanowires, nanobelts, and nanosheets. The Raman spectra of nanobelts and nanosheets are notably different from those of nanowires, exhibiting a strong enhancement of the multiphonon response. Moreover, the first-order longitudinal optical (LO) phonon energy systematically increases with increasing lateral size from nanowires to nanobelts, and to nanosheets. These results suggest that the optical phonons in the CdS nanostructures are influenced by strain, crystallinity, and exciton-LO phonon coupling. (C) 2007 American Institute of Physics. [DOI: 10.1063/1.2806937]
\end{abstract}

Nanowire-based nanostructures enable one to design novel optoelectronic devices such as nanoscale lasers ${ }^{1}$ and polarization-sensitive photodetectors. ${ }^{2}$ More recently, CdS nanostructures have attracted considerable attention. ${ }^{3,4} \mathrm{Re}-$ cent progress in growth techniques which control growth parameters makes possible the synthesis not only of onedimensional nanowires (NWs) but also of two-dimensional nanobelts (NBs) and nanosheets (NSs), providing even more diverse possibilities for optoelectronic applications. ${ }^{5-8}$

The optical properties of nanostructures are influenced by size confinement, ${ }^{9}$ strain, ${ }^{7,10-12}$ crystallinity ${ }^{7}$ and excitonlongitudinal optical (LO) phonon interaction. ${ }^{11,13-15}$ For instance, the LO phonon energies of CdS NWs with diameters larger than $50 \mathrm{~nm}$ do not exhibit quantum confinement effects and are mostly affected by lattice contraction. ${ }^{9,11,12}$ Lattice contraction can be induced by surface tension during surface reconstruction in the growth of CdS NWs (Refs. 11 and 12) and CdSe NBs, ${ }^{7}$ resulting in a significant increase of the LO phonon energy. Good crystallinity provides for welldefined and long-lived phonons which may enhance multiphonon responses. For instance, Venugopal et al. have observed that the multiphonon responses of CdSe NBs are much stronger than those of bulk CdSe source powder, suggesting an improvement in crystallinity in NBs. ${ }^{7}$ Moreover, an enhancement of the second-order LO (2LO) phonon response can be indicative of an increased exciton-LO phonon coupling strength. ${ }^{11,13}$ By evaluating the intensity ratio of the 2LO to first-order LO (1LO) phonon responses, Shiang et al. have shown that the exciton-LO phonon coupling strength in $\mathrm{CdS}$ quantum dots increases with increasing dot size. ${ }^{13}$ Theory also predicts that the exciton-LO phonon coupling strength is larger in bulk than in quantum dots or NWs. 14,15 In contrast, Pan et al. observed that the $2 \mathrm{LO}$ phonon response is enhanced in CdS NW compared to bulk CdS, suggesting that the exciton-LO phonon coupling strength in the NW is much stronger than that in the bulk. ${ }^{11}$ Thus, the size dependence of the exciton-LO phonon coupling strength in the CdS nanostructures remains unsettled. In this letter, we report Raman scattering data from $\mathrm{CdS}$ nanostructures in order to understand how strain, crystallinity, and exciton-LO

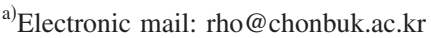

phonon coupling evolve as the lateral size is increased from NWs to NBs and, finally, to NSs.

$\mathrm{CdS}$ nanostructures were grown by pulsed laser deposition using vapor-phase transport of source vapor onto a $\mathrm{Au}$ film which was deposited on the Si substrates. Various types of nanostructures including NWs, NBs, and NSs were obtained with a processing temperature in the range of $600-800{ }^{\circ} \mathrm{C}$ and with a laser energy density in the range of
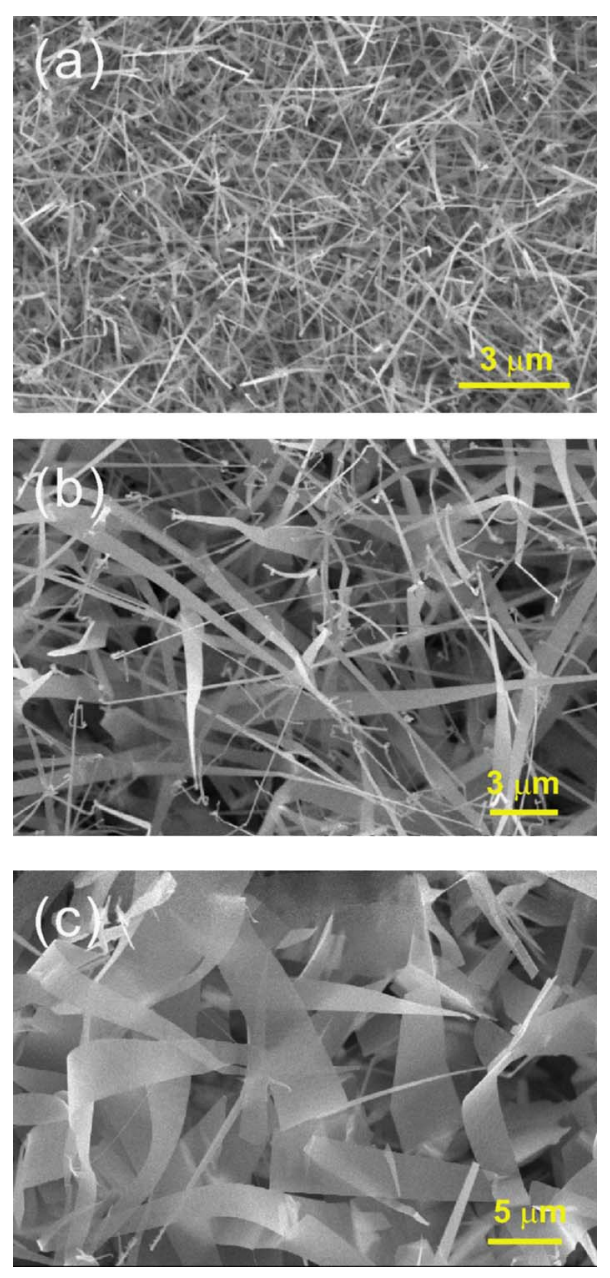

FIG. 1. (Color online) SEM images of CdS (a) nanowires, (b) nanobelts, and (c) nanosheets. 

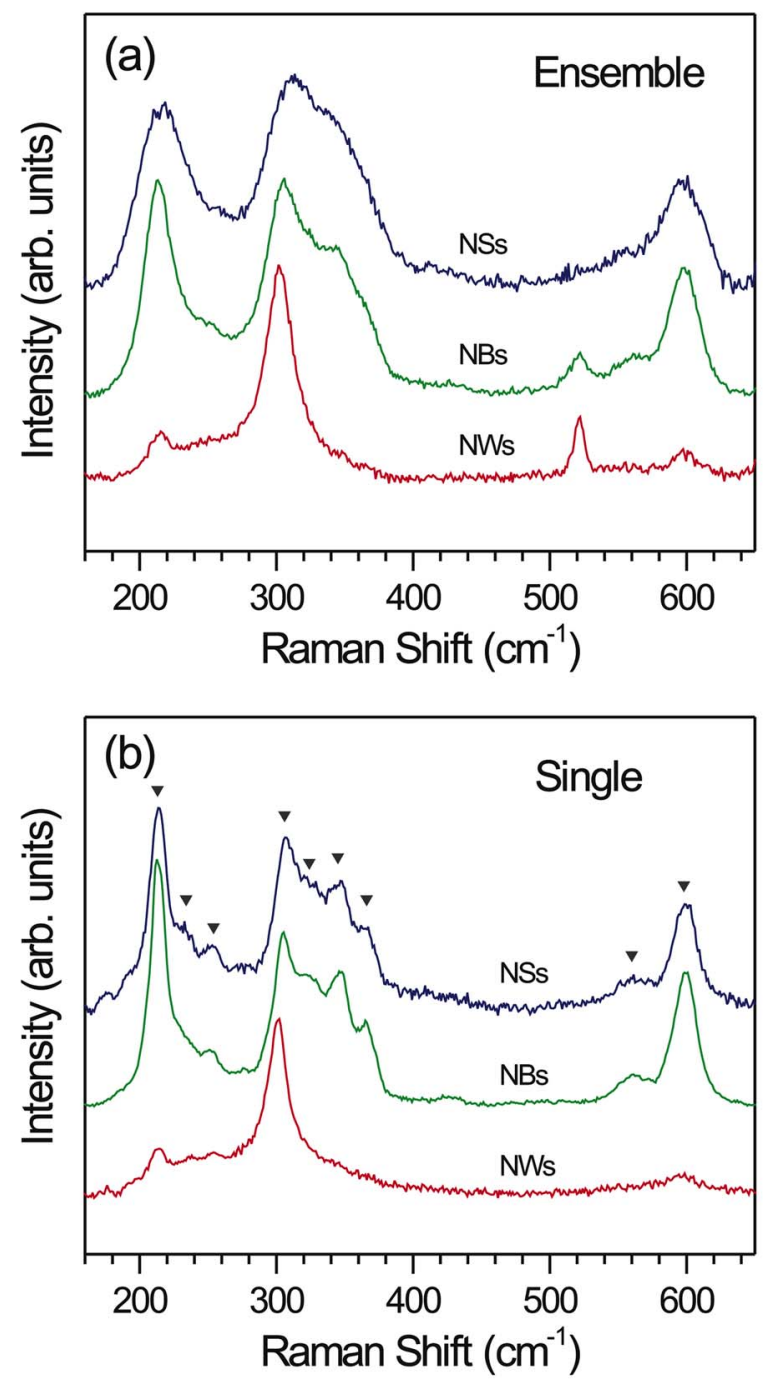

FIG. 2. (Color online) Raman spectra from (a) ensemble and (b) single CdS nanowires, nanobelts, and nanosheets.

$1-5 \mathrm{~J} / \mathrm{cm}^{2}$. Raman scattering measurements were performed at room temperature using a $\mathrm{He}-\mathrm{Ne}$ laser with an excitation wavelength of $633 \mathrm{~nm}$ in a backscattering geometry. Incident light was focused on a sample surface through an optical microscope with a spatial resolution of $\sim 2$ or $\sim 1 \mu \mathrm{m}$ for ensemble or single samples, respectively. The scattered light from the samples was dispersed through a monochromator and was recorded using a thermoelectrically cooled chargecoupled device detector. For single nanostructure measurements, each of the as-grown samples was put into an ethanol solution, sonicated in order to detach single nanostructures from the substrate, and dispersed onto a separate cover-glass plate.

Figures 1(a)-1(c) show the representative scanning electron microscope (SEM) images of CdS NWs, NBs, and NSs, respectively. NW diameters are in the range of 50-200 nm. The typical thickness of the NBs and NSs is $50 \mathrm{~nm}$. The typical width of the NBs is in the range of $0.5-1.5 \mu \mathrm{m}$, but that of the NSs is much wider, extending up to $5 \mu \mathrm{m}$. X-ray diffraction, selective area electron diffraction, and transmission electron microscope results have revealed the synthesized CdS nanostructures to be wurtzite-type single crystals. 16

Figure 2(a) shows the Raman spectra from ensemble

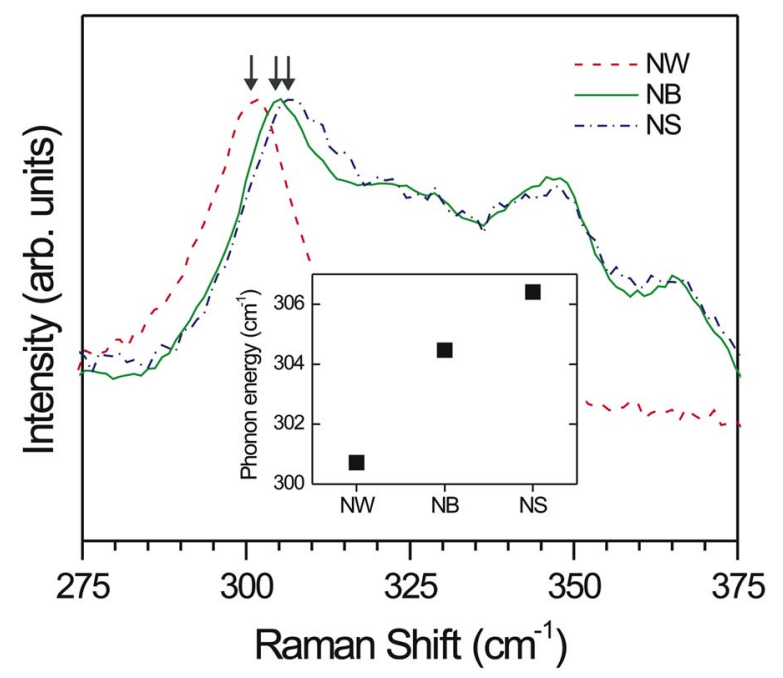

FIG. 3. (Color online) Raman spectra from a single CdS nanowire, nanobelt, and nanosheet. The inset shows the $A_{1} 1 \mathrm{LO}$ phonon energies of the individual CdS nanostructures.

CdS NWs, NBs, and NSs. $A_{1}, E_{1}$, and $E_{2}$ symmetry modes are allowed for wurtzite CdS (space group $C_{6 v}^{4}-C 6_{3} m c$ ). The Raman modes at 301 and $598 \mathrm{~cm}^{-1}$ observed in the ensemble NWs correspond to the $A_{1} 1 \mathrm{LO}$ and $2 \mathrm{LO}$ phonons, respectively, and are similar to results observed by others. ${ }^{8,9,11-13,17,18}$ A weak response at $213 \mathrm{~cm}^{-1}$ and a broad response around $240 \mathrm{~cm}^{-1}$ are also observed. Note that the Raman peak at $521 \mathrm{~cm}^{-1}$ observed in the ensemble NWs is due to the Si substrate. The Raman spectra of the ensemble CdS NBs and NSs are considerably different from those of the ensemble CdS NWs with the $213 \mathrm{~cm}^{-1}$ and $2 \mathrm{LO}$ modes considerably enhanced. Moreover, additional broad modes appear around $340 \mathrm{~cm}^{-1}$ and at $560 \mathrm{~cm}^{-1}$. Notably, the $1 \mathrm{LO}$ phonon energy is seen to shift upward systematically as the lateral size increases from NWs to NBs to NSs. These optical phonon behaviors are related to the strain, crystalline quality, and exciton-LO phonon coupling in the CdS nanostructures.

Raman spectroscopy of single NWs, NBs, or NSs has advantages over an ensemble measurement. Figures 2(a) and 2(b) clearly show noticeable differences between the single and ensemble spectra. The phonon linewidths are much narrower in the single spectra than in the corresponding ensemble ones. The broadening of the phonon line shapes in the ensemble spectra suggests an inhomogeneity in strain due to size and shape fluctuations of individual nanostructures within the area sampled. In the NS data, the broad response observed around $240 \mathrm{~cm}^{-1}$ in the ensemble spectra is seen to consist of two phonon modes at 233 and $253 \mathrm{~cm}^{-1}$ in the single spectra. The broad response around $340 \mathrm{~cm}^{-1}$ in the ensemble spectra is revealed in more detail as the 324 , 346 , and $366 \mathrm{~cm}^{-1}$ phonon modes in the spectra from single NSs.

Figure 3 summarizes the systematic upward shifts of the $1 \mathrm{LO}$ phonon energy. The $1 \mathrm{LO}$ phonon mode positions were extracted by fitting these modes to a Lorentzian line shape. Earlier studies of CdS NWs reported that the 1LO phonon energy was shifted upward by $5 \mathrm{~cm}^{-1}$ compared to that of bulk CdS, suggesting that the lattice contraction of the $c$ axis is induced during the NW growth. ${ }^{11,12}$ The $A_{1} 1 \mathrm{LO}$ phonon is related to vibrations along the $c$ axis. ${ }^{10,19}$ Here, we observe the $1 \mathrm{LO}$ phonon energy increases systematically by $\sim 6 \mathrm{~cm}^{-1}$ 
from a single NW ( $\sim 200 \mathrm{~nm}$ diameter $)$ to a single NB ( $\sim 1.3 \mu \mathrm{m}$ width) and to a single NS ( $\sim 3.4 \mu \mathrm{m}$ width), suggesting that the lattice contracts as the lateral size increases.

The strain associated with a lattice contraction of $\Delta c / c$ can be calculated using the following equation; $\Delta \omega / \omega_{0}$ $=(1+3 \Delta c / c)^{-\gamma}-1$, where $\Delta \omega$ is the 1LO phonon energy shift from its bulk value $\omega_{0}$ and $\gamma$ is the Grüneisen parameter (1.1 for CdS $).{ }^{10,20}$ The downward shift of the 1LO phonon energy due to size confinement is negligible for the $\mathrm{CdS}$ NWs with diameters larger than $50 \mathrm{~nm}$. ${ }^{9}$ For the lattice contraction of the single NB and NS compared to the single NW, we obtain $\Delta c / c=-0.37 \%$ and $-0.56 \%$ for the single CdS NB and NS, respectively. For both the freestanding $\mathrm{CdSe}$ nanocrystals ${ }^{10}$ and the $\mathrm{CdS}_{1-x} \mathrm{Se}_{x}$ nanocrystals embedded in glass, ${ }^{20}$ the lattice contraction was reported to be larger for smaller nanocrystals. In contrast, our results indicate that the lattice contraction increases as you move to larger nanostructures, from CdS NWs to NBs to NSs.

The Raman modes we observe at 213, 324, 346, 366, and $560 \mathrm{~cm}^{-1}$ are attributed to additional multiphonon responses. Similar multiphonon responses were observed in bulk CdS at 207, 328, 347, 364, and $556 \mathrm{~cm}^{-1}{ }^{18}$ Tell et al. have identified the $A_{1}$ transverse optical (TO), $E_{1}$ TO, and $E_{2}$ modes at 228, 235, and $252 \mathrm{~cm}^{-1}$, respectively. ${ }^{18}$ Therefore we attribute the $233 \mathrm{~cm}^{-1}$ phonon response to the $A_{1}$ TO mode, $E_{1}$ TO mode, or their combination. The $253 \mathrm{~cm}^{-1}$ mode corresponds to the $E_{2}$ phonon mode. These assignments are in good agreement with those of Fu et al. ${ }^{12}$

The strength of the multiphonon responses also provides useful information on crystalline quality ${ }^{7}$ and exciton-LO phonon coupling. ${ }^{11,13}$ For instance, multiphonon responses are enhanced in CdSe NBs compared to bulk CdSe, suggesting that the NBs have better crystalline quality. ${ }^{7}$ Similarly, we suggest that the significant enhancement of the multiphonon responses in the CdS NBs and NSs is indicative of better crystalline quality in these materials than in the NWs. Pan et al. have observed that the intensity ratio of the $2 \mathrm{LO}$ to $1 \mathrm{LO}$ phonon responses is much larger in CdS NWs than in bulk CdS, suggesting that the exciton-LO phonon coupling strength is much larger in the CdS NWs. ${ }^{11}$ In our study, the 2LO phonon response shown in Fig. 2 is much stronger in both the CdS NBs and NSs compared to the CdS NWs, suggesting that the exciton-LO phonon coupling is strengthened for the larger sized CdS nanostructures.

In summary, we report Raman spectra from high quality CdS NWs, NBs, and NSs both in ensemble form and as single nanostructures. We observe that Raman spectra exhibit a strong dependence on lateral size. The NWs, NBs, and NSs display a systematic increase of the 1LO phonon energy, indicating that a lattice contraction occurs with increasing lateral size. Moreover, the observation of a strong multiphonon response suggests enhanced crystallinity and/or strengthened exciton-LO phonon coupling in both CdS NBs and NSs.

The authors thank Professor Howard E. Jackson and Professor Jan Yarrison-Rice for their valuable comments. This work was supported by the Korea Research Foundation Grant funded by the Korean Government (MOEHRD) (KRF-2006-312-C00183). The work at KIST was supported by the KIST-CNRS LIA (2U03450) program.

${ }^{1}$ M. H. Huang, S. Mao, H. Feick, H. Yan, Y. Wu, H. Kind, E. Weber, R. Russo, and P. Yang, Science 292, 1897 (2001).

${ }^{2}$ J. Wang, M. S. Gudiksen, X. Duan, Y. Cui, and C. M. Lieber, Science 293, 1455 (2001).

${ }^{3}$ X. Duan, Y. Huang, R. Agarwal, and C. M. Lieber, Nature (London) 421, 241 (2003).

${ }^{4}$ R. Agarwal, C. J. Barrelet, and C. M. Lieber, Nano Lett. 5, 917 (2005).

${ }^{5}$ Z. R. Dai, Z. W. Pan, and Z. L. Wang, Adv. Funct. Mater. 13, 9 (2003).

${ }^{6}$ J.-H. Park, H.-J. Choi, Y.-J. Choi, S.-H. Sohn, and J.-G. Park, J. Mater. Chem. 14, 35 (2004).

${ }^{7}$ R. Venugopal, P. I. Lin, C. C. Liu, and Y. T. Chen, J. Am. Chem. Soc. 127, 11262 (2005).

${ }^{8}$ Z. Q. Wang, J. F. Gong, J. H. Duan, H. B. Huang, S. G. Yang, X. N. Zhao, R. Zhang, and Y. W. Du, Appl. Phys. Lett. 89, 033102 (2006).

${ }^{9}$ D. Routkevitch, T. L. Haslett, L. Ryan, T. Bigioni, C. Douketis, and M. Moskovits, Chem. Phys. 210, 343 (1996).

${ }^{10}$ J.-Y. Zhang, X.-Y. Wang, M. Xiao, L. Qu, and X. Peng, Appl. Phys. Lett. 81, 2076 (2002)

${ }^{11}$ A. Pan, R. Liu, Q. Yang, Y. Zhu, G. Yang, B. Zou, and K. Chen, J. Phys. Chem. B 109, 24268 (2005).

${ }^{12}$ X. L. Fu, L. H. Li, and W. H. Tang, Solid State Commun. 138, 139 (2006).

${ }^{13}$ J. J. Shiang, S. H. Risbud, and A. P. Alivisatos, J. Chem. Phys. 98, 8432 (1993).

${ }^{14}$ W. Z. Shen, Appl. Phys. Lett. 79, 1285 (2001).

${ }^{15}$ W. Z. Shen, Physica B 322, 201 (2002).

${ }^{16}$ H. Rho, K.-Y. Lee, T. B. Hoang, L. V. Titova, A. Mishra, L. M. Smith, H. E. Jackson, J. M. Yarrison-Rice, Y.-J. Choi, K.-J. Choi, and J.-G. Park (unpublished).

${ }^{17}$ A. Abdi, L. V. Titova, L. M. Smith, H. E. Jackson, J. M. Yarrison-Rice, J. L. Lensch, and L. J. Lauhon, Appl. Phys. Lett. 88, 043118 (2006).

${ }^{18}$ B. Tell, T. C. Damen, and S. P. S. Porto, Phys. Rev. 144, 771 (1966).

${ }^{19}$ O. Zelaya-Angel, F. de L. Castillo-Alvarado, J. Avendaño-López, A. Escamilla-Esquivel, G. Contreras-Puente, R. Lozada-Morales, and G. Torres-Delgado, Solid State Commun. 104, 161 (1997).

${ }^{20}$ G. Scamarcio, M. Lugará, and D. Manno, Phys. Rev. B 45, 13792 (1992). 